\title{
APLICAÇÃO DE TÉCNICAS ESTATÍSTICAS MULTIVARIADAS PARA ESTUDOS DA ATIVIDADE PECUÁRIA NA REGIÃO ADMINISTRATIVA DE PRESIDENTE PRUDENTE
}

Juliana Andréa Osorio Balan ${ }^{1}$, Antônio Assiz de Carvalho Filho ${ }^{2}$, Ricardo da Fonseca ${ }^{3}$, Eduardo Cardozo de Oliveira ${ }^{4}$.

${ }^{1}$ Graduanda em estatística, UNESP - Presidente Prudente. ${ }^{2}$ Docente do departamento de estatística, UNESP - Presidente Prudente. ${ }^{3}$ Docente do curso de Zootecnia, UNESP - Dracena. ${ }^{4}$ Mestrando do programa matemática aplicada e computacional, UNESP - Presidente Prudente.

\section{RESUMO}

O objetivo deste trabalho foi conhecer a estrutura produtiva da pecuária de corte na região administrativa de Presidente Prudente. Neste trabalho foram utilizados dois tipos de análise estatística multivariada: as técnicas de interdependência de análise fatorial e análise de agrupamento. Pretendeu-se contribuir para o aprofundamento do conhecimento da realidade regional, com o intuito de fornecer subsídios aos pesquisadores que tenham como meta elaborar políticas públicas visando à melhoria e o crescimento no âmbito pecuário dessa região.

Palavras-chave: políticas públicas; análise de componentes principais; análise fatorial; análise de agrupamento.

\section{MULTIVARIATE STATISTICAL TECHNIQUES APPLICATION TO STUDIES OF LIVESTOCK ACTIVITY IN THE ADMINISTRATIVE REGION OF PRESIDENTE PRUDENTE.}

\begin{abstract}
The objective of this study is to know the productive structure of beef cattle in the administrative region of Presidente Prudente. In this work we used two types of multivariate statistical analysis: techniques interdependence of factor analysis is and cluster analysis. It is intended to contribute to a deeper understanding of the regional reality in order to provide grants to researchers who have a go al to develop public politics proving live stock and grow thin this region.
\end{abstract}

Keywords: public politics; principal component analysis; factor analysis; cluster analysis. 


\section{INTRODUÇÃO}

O Estado de São Paulo é reconhecido nacionalmente como a unidade da Federação com melhores índices de desenvolvimento urbano e rural. Apresenta a maior densidade demográfica do país e abriga a mais complexa região metropolitana. Tem importância fundamental para a economia do Brasil ao responder por parte expressiva do Produto Interno Bruto. Sua participação é representativa na produção agropecuária, industrial e no setor terciário. Além disso, é o principal centro exportador nacional (FIRETTI et al., 2010).

Visando aperfeiçoar o planejamento político-administrativo por parte do Governo Estadual, São Paulo foi dividido no ano de 1967, em 15 Regiões Administrativas (incluindo a Região Metropolitana), sendo algumas delas subdivididas em Regiões de Governo. Segundo Silva (1995) esta regionalização foi feita considerando-se apenas os aspectos locais, com aglomerados de municípios circunvizinhos, sem levar em consideração características econômicas ou sociais.

A 10a Região Administrativa do Estado de São Paulo, cuja sede é o município de Presidente Prudente é considerada uma das últimas fronteiras do desenvolvimento paulista, com presença constante nos noticiários que tratam de conflitos pela posse de terras, e resultado da expansão descontrolada que constitui um empecilho considerável ao progresso local.

O objetivo deste trabalho foi conhecer a estrutura produtiva da pecuária de corte na Região Administrativa (RA) de Presidente Prudente, localizada na região oeste do Estado de São Paulo. Pretendeu-se contribuir para o aprofundamento do conhecimento da realidade regional, com o intuito de fornecer subsídios aos pesquisadores que tenham como meta elaborar políticas públicas visando à melhoria e o crescimento no âmbito pecuário da região.

\section{METODOLOGIA}

A escolha das variáveis foi realizada de acordo com o objetivo principal do trabalho que foi analisar a estrutura produtiva da pecuária de corte da RA de Presidente Prudente, envolvendo assim todos os ruminantes voltados para a pecuária de corte, suas respectivas unidades de produção agrícola (UPA) destinadas à produção dos mesmos e o valor adicionado à agropecuária.

As variáveis foram coletadas da base de dados oficiais do Projeto LUPA (Levantamento Censitário das Unidades de 
Produção Agropecuária do Estado de São Paulo, www.cati.sp.gov.br) e da Fundação SEADE, ambas referentes aos anos de 2007/2008. Colocou-se em uma planilha Excel, - levantamento e a coleta dos dados referentes a cada variável correspondente aos 53 municípios que compõem a RA de Presidente Prudente.

As variáveis de escolha foram:

- número de cabeça de bovinocultura mista.

- número de cabeça de bovinocultura de corte.

- número de cabeça de bubalinocultura.

- número de cabeça de caprinocultura.

- número de cabeça de ovinocultura.

- número de unidades de produção agrícola destinada à produção de bovinocultura mista.

- número de unidades de produção agrícola destinada à produção de bovinocultura de corte.

- número de unidades de produção agrícola destinada à produção de bubalinocultura.

- número de unidades de produção agrícola destinada à produção de caprinocultura.

- número de unidades de produção agrícola destinada à produção de ovinocultura.

- Valor adicionado à agropecuária referente a cada município, em 2008.

Foram utilizadas para análise dos dados duas técnicas estatísticas multivariadas, utilizando o programa estatístico R.
Segundo Hair et al. (2009), análise multivariada se refere a todas as técnicas estatísticas que simultaneamente analisam múltiplas medidas sobre indivíduos ou objetos sob investigação. Assim, qualquer análise simultânea de mais do que duas variáveis podem ser consideradas, a princípio, como multivariada.

Neste trabalho foram utilizados dois tipos de análise estatística multivariada: as técnicas de interdependência de análise fatorial e análise de agrupamento. A seguir será abordada cada uma das técnicas.

\subsection{Análise Fatorial}

A análise fatorial é uma técnica de interdependência, cujo propósito principal é definir a estrutura inerente entre as variáveis na análise. A análise fatorial fornece ferramentas para analisar a estrutura das inter-relações (correlações) em um grande número de variáveis definindo conjuntos de variáveis que são fortemente interrelacionadas, conhecidos como fator. O propósito geral da técnica de análise fatorial é encontrar um modo de resumir a informação contida em diversas variáveis originais em um conjunto menor de novas variáveis estatísticas, que são os fatores, com perda mínima de informação (HAIR et al., 2009). 
De acordo com Mingoti (2005), a análise fatorial é uma técnica estatística multivariada que vem sendo muito utilizada em diversas áreas atualmente.

Ainda, a análise fatorial subdivide-se em dois grupos: análise fatorial $\mathrm{R}$ e análise fatorial Q. A análise fatorial $\mathrm{R}$ é o tipo mais comum de análise fatorial, analisa um conjunto de variáveis para identificar os fatores. Já a análise fatorial $Q$ não é utilizada muito freqüentemente por causa das dificuldades computacionais. Em vez disso, a maioria dos pesquisadores utiliza a análise de agrupamento cujo objetivo é classificar uma amostra de entidades em um número menor de grupos com base nas suas similaridades (HAIR et al., 2009). Para este trabalho foram selecionadas a análise fatorial $\mathrm{R}$ e a análise de agrupamento.

\subsubsection{Suposições para a Aplicação da análise} Fatorial

Hair et al. (2009) dizem que as suposições críticas na análise fatorial são muito mais conceituais do que estatísticas. Em relação às questões estatísticas temos que apenas garantir que a matriz de dados tenha correlações suficientes para justificar a aplicação da análise fatorial, assim, neste trabalho empregou-se o teste de medição de adequação da amostra (MSA), do inglês Measure Sampling Adequacy e o índice varia entre $0 \leq M S A \leq 1$, sendo 10 valor ideal. $A$ medida pode ser interpretada com as seguintes orientações: 0,80 ou acima, admirável; 0,70 ou acima, mediano; 0,60 ou acima, medíocre; 0,50 ou acima, ruim; e abaixo de 0,50, inaceitável. O pesquisador deve examinar os valores MSA para cada variável e excluir aquelas que estão no domínio inaceitável. No processo de eliminação das variáveis, o pesquisador deve primeiro eliminar a variável com o menor MSA e então recalcular a análise fatorial. Deve-se continuar o processor de eliminar a variável com o menor MSA abaixo de 0,50 até que todas as variáveis tenham um valor aceitável. Uma vez que as variáveis individuais atinjam um nível aceitável, então o MSA geral pode ser calculado e uma decisão pode ser tomada sobre a continuidade da análise fatorial.

Então depois que as variáveis forem especificadas e a matriz de correlação amostral preparada, aplicamos a análise fatorial.

\subsubsection{Determinação de Fatores}

Para determinar o número de fatores utilizou-se o método das componentes principais e para estimar o número desses 
fatores utilizamos três critérios que a seguir foram abordados.

\subsubsection{Métodos das Componentes Principais}

O método das componentes principais é um dos mais comumente utilizados para extração dos fatores e consiste em estimarmos uma matriz resultante baseada nos autovalores e autovetores extraídos da matriz de correlação amostral. Este método por si só, é uma excelente técnica exploratória de dados multivariados, podendo assim, ser combinada com outras técnicas estatísticas multivariadas para resultados mais precisos e claros (MINGOTI, 2005).

A análise de componentes principais consiste em explicar a estrutura da variância de combinações lineares criadas a partir das variáveis originais. Podemos dizer que os objetivos dessa técnica são basicamente a redução de dados e a interpretação dos mesmos (JOHNSON; WICHERN, 1998).

Outro ponto interessante citado por Johnson e Wichern (1998) foi que a análise de componentes principais geralmente revela relações e interpretações que não eram possíveis de serem vistas antes da aplicação da técnica. O autor também cita que a suposição de normalidade não é um requisito necessário para a aplicação desta técnica.

\subsubsection{Estimação do Número de Fatores}

Uma vez que se possuiu a matriz de correlação amostral, basta extrairmos os autovalores dessa matriz e ordená-los em ordem decrescente. Depois de feita a ordenação, alguns critérios são descritos por Hair et al. (2009) para a escolha do número de fatores:

Critério da raiz latente: Os fatores que possuíram os autovalores maiores que 1 são considerados significantes, logo todos os fatores que são menores que 1 são descartados.

Critério de percentagem da variância: Assim como na análise de componentes principais, pré-estabelecemos um percentual de corte para a variância total explicada e, ao atingir esse valor especificado, retemos os fatores.

Critério do teste scree: Assim como na análise de componentes principais, para retermos o número de fatores, basta observarmos no gráfico o ponto onde os valores obtidos para cada autovalor tende a se estabilizar, então o número de autovalores anteriores a esse ponto, será o número de fatores.

$\mathrm{Na}$ prática, normalmente utilizam-se mais de um critério para a escolha do número de fatores. 


\subsubsection{Rotação de Fatores}

Os valores observados na matriz fatorial são chamados de cargas fatoriais. As cargas fatoriais, nada mais são do que a correlação de cada variável com um determinado fator, ou seja, elas indicam o grau de correspondência entre a variável e o fator, sendo as cargas numericamente maiores responsáveis pela denominação que o fator receberá.

Quando obtiver os fatores por meio de um ou mais critérios citados anteriormente, atingiu-se o objetivo de resumir os dados em novas variáveis aleatórias, porém na maioria dos casos, os fatores sem rotação não nos fornecem informação necessária para uma interpretação adequada das variáveis. Nesse caso temos que utilizar um recurso de transformação desses fatores. Em outras palavras, quando uma variável possui correlações com grandezas numericamente similares em mais de um fator, a interpretação desses fatores se torna algo muito complexo. A rotação é desejável, pois ela simplifica a estrutura fatorial, tornando mais fácil a interpretação das novas variáveis; ou seja, com a rotação dos fatores, conseguiu-se soluções mais simples e teoricamente mais significativas. Logo, pode-se dizer que a rotação de fatores é uma importante ferramenta na interpretação dos mesmos (HAIR et al., 2009).

\subsubsection{Rotação Ortogonal de Fatores}

A rotação fatorial consiste em rotacionar os eixos de referências dos fatores, até que uma posição seja alcançada. O efeito final dessa rotação é redistribuir a variância dos fatores para atingir um padrão fatorial mais simples.

Utilizamos o método Varimax para realizar a rotação ortogonal de fatores. 0 método Varimax concentra-se na simplificação das colunas da matriz fatorial, ou seja, o método tem por objetivo maximizar as cargas fatoriais das variáveis associadas a apenas um fator. O método Varimax vem sendo o mais utilizado dentre todos os outros métodos para rotação ortogonal de fatores, pois segundo a literatura estudada, é o método que produz as soluções mais simples (HAIR et al., 2009).

\subsubsection{Nomeação dos Fatores}

Em soluções ortogonais, os fatores são independentes um do outro. Portanto, devemos estudar cada fator separadamente. As variáveis que possuem cargas fatoriais significativas para determinado fator, são as que mais contribuem com a rotulação do mesmo. 
Em determinado fator, pode haver diversas variáveis com cargas elevadas. Neste caso tenta-se nomear o fator de modo que esse rótulo reflita todas essas variáveis com cargas significativas; esse rótulo tem como objetivo, impor um significado para esse fator.

O rótulo dado para o fator depende, única e exclusivamente, da sensibilidade do pesquisador, uma vez que os pacotes computacionais não fornecem esses nomes (HAIR et al., 2009).

\subsection{Análise de Agrupamento}

A análise de agrupamentos é uma técnica analítica para desenvolver subgrupos significativos. Especificamente, o objetivo é classificar uma amostra de entidades em um número menor de grupos com base nas suas similaridades. Geralmente envolve pelo menos três passos. O primeiro é a medida de alguma forma de similaridade ou associação entre as entidades para determinar quantos grupos realmente existem na amostra. O segundo passo é o real processo de agrupamento, onde as entidades são particionadas em grupos (agrupamentos). O último passo é estabelecer - perfil das variáveis para determinar sua composição (HAIR et al., 2009).

\section{RESULTADOS}

\subsection{Análises Gráficas dos Dados}

Na Figura 1, é apresentada a matriz de dispersão das variáveis. Os valores acima da diagonal principal são as correlações bivariadas; abaixo da diagonal principal observamos os gráficos de dispersão; e a diagonal principal representa a distribuição de cada variável, na forma de um histograma. 


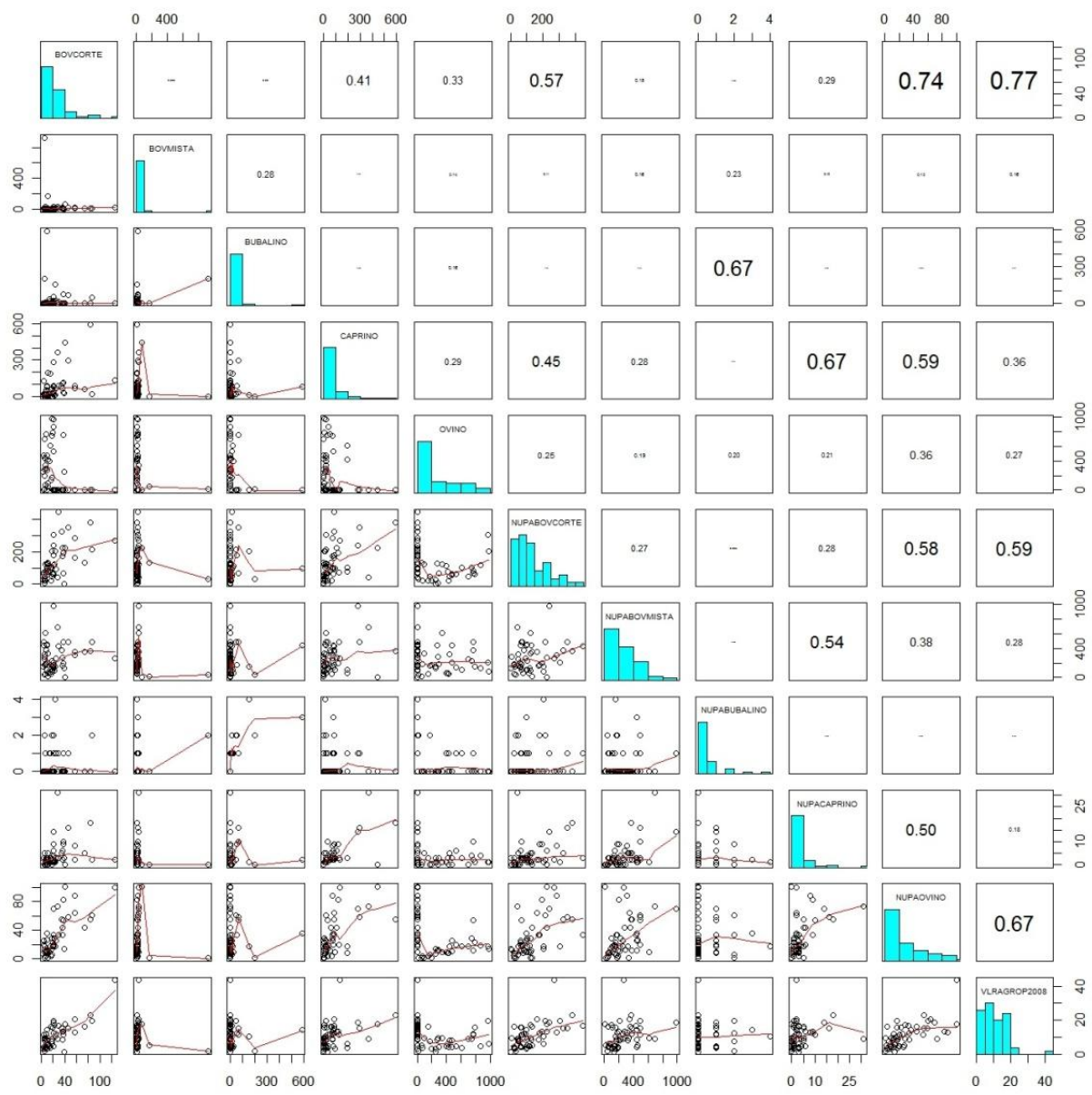

Figura 1. Matriz de dispersão das variáveis em estudo na R.A. de Presidente Prudente.

As correlações significativas com valores acima de 0,5 são: 0,77 de correlação entre a bovinocultura de corte e o valor adicionado da agropecuária em 2008, 0,74 de correlação entre a bovinocultura de corte e o número de UPA com ovinocultura, 0,69 de correlação entre o número de UPA com ovinocultura e o valor adicionado da agropecuária em 2008, 0,59 de correlação entre a caprinocultura e o número de UPA com ovinocultura, 0,59 de correlação entre o número de UPA com bovinocultura de corte e o valor adicionado da agropecuária em 2008, 0,58 de correlação entre o número de UPA com bovinocultura de corte e o número de UPA com ovinocultura, 0,54 de correlação entre a bovinocultura mista e o número de UPA com caprinocultura, 0,50 de correlação entre o número de UPA com caprinocultura e o número de UPA com ovinocultura. 
Pode-se afirmar que a produção de bovinos de corte e ovinos contribui significativamente para a economia da região. A produção de bovinos de corte, em cabeças e o número de propriedades destinadas à produção de ovinos estão fortemente correlacionadas. Já a produção de caprinos aparece como variável surpresa quando vista correlacionadamente com o número de propriedades destinadas à produção de ovinos e com a produção de bovinos mistos.

Na figura 2, apresenta-se o gráfico Two Way Joining, este é um gráfico de agrupamento.

O gráfico mostra uma correlação significativa entre: o município de Rancharia com a produção de bovinos destinados a corte e com o valor adicionado da agropecuária em 2008; o município de Adamantina com a produção de bubalinos; o município de Pracinha com a produção bovinocultura mista; o município de Martinópolis com a produção de caprinos; o município de Junqueirópolis com a produção de ovinos; o município de Anhumas com o número de propriedades com bovinocultura mista; o município de Euclides da Cunha Paulista com o número de propriedades destinadas à produção de Bubalinos e o município de Presidente Prudente com o número de propriedades com bovinocultura de corte.

\subsection{Aplicações das Técnicas nas Variáveis de Produção}

Na tabela 1, apresenta-se os valores do teste MSA para prosseguir com a aplicação das técnicas da análise fatorial.

Tabela 1. Aplicação do teste MSA nas variáveis de produção.

\begin{tabular}{ll}
\hline VARIÁVEIS & VALORES \\
\hline Bovinocultura de corte & 0,66 \\
\hline Bovinocultura mista & 0,56 \\
\hline Bubalinocultura & 0,52 \\
\hline Ovinocultura & 0,75 \\
\hline Caprinocultura & 0,53 \\
\hline
\end{tabular}

Os valores do teste MSA variam entre 0 $\leq \mathrm{MSA} \leq 1$, sendo 1 o valor ideal e acima de 0,5 os valores são aceitáveis para prosseguirmos com a análise fatorial. 
ESTUDO ESTATÍSTICO DA PECUÁRIA NA REGIÃO DE PRESIDENTE PRUDENTE.

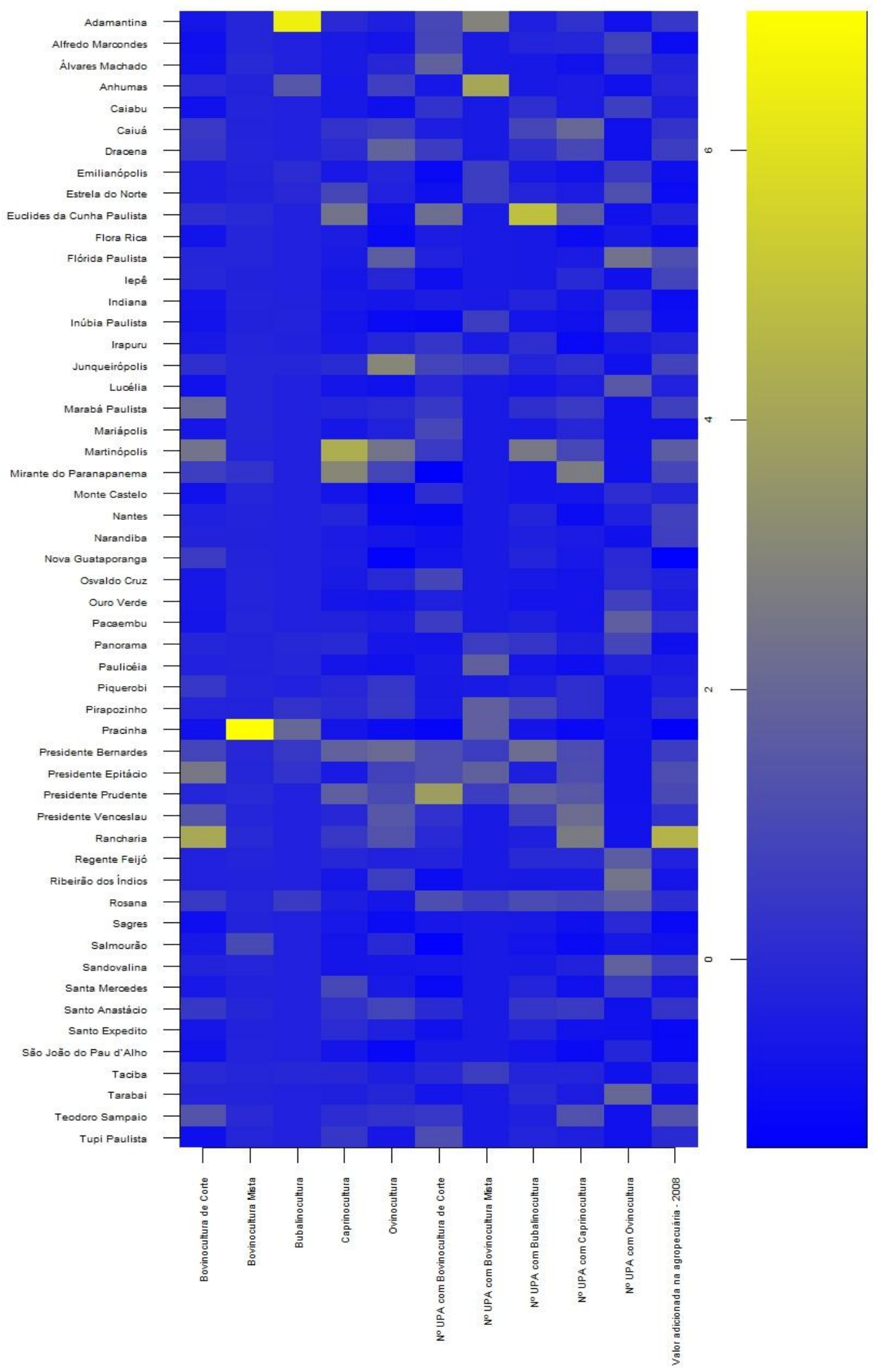

Figura 2. Gráfico de agrupamento entre os municípios e as variáveis em estudo. 
A tabela 2 apresenta os valores (valores acima de um) como significativos e os correspondentes aos autovalores para as demais valores foram excluídos. Analisando o variáveis de produção.

critério de percentagem da variância considera-se que os dois primeiros valores Tabela 2. Lista de autovalores para as variáveis de produção.

\begin{tabular}{lll}
\hline & AUTOVALORES & VARIÂNCIA \% \\
\hline 1. & 2.00141 & 40,03 \\
\hline 2. & 1.23691 & 24,74 \\
\hline 3. & 0.72416 & 14,48 \\
\hline 4. & 0.61593 & 12,32 \\
\hline 5. & 0.42159 & 8,43 \\
\hline
\end{tabular}

Analisando o critério da raiz latente possuem $64,77 \%$ da variância explicada e descarta-se os demais valores.

O terceiro critério utilizado foi o teste de scree. Observamos na Figura 3 o ponto onde os valores obtidos para cada autovalor tende a se estabilizar, então o número de autovalores anteriores a esse ponto, será o número de fatores. considerou-se os dois primeiros valores

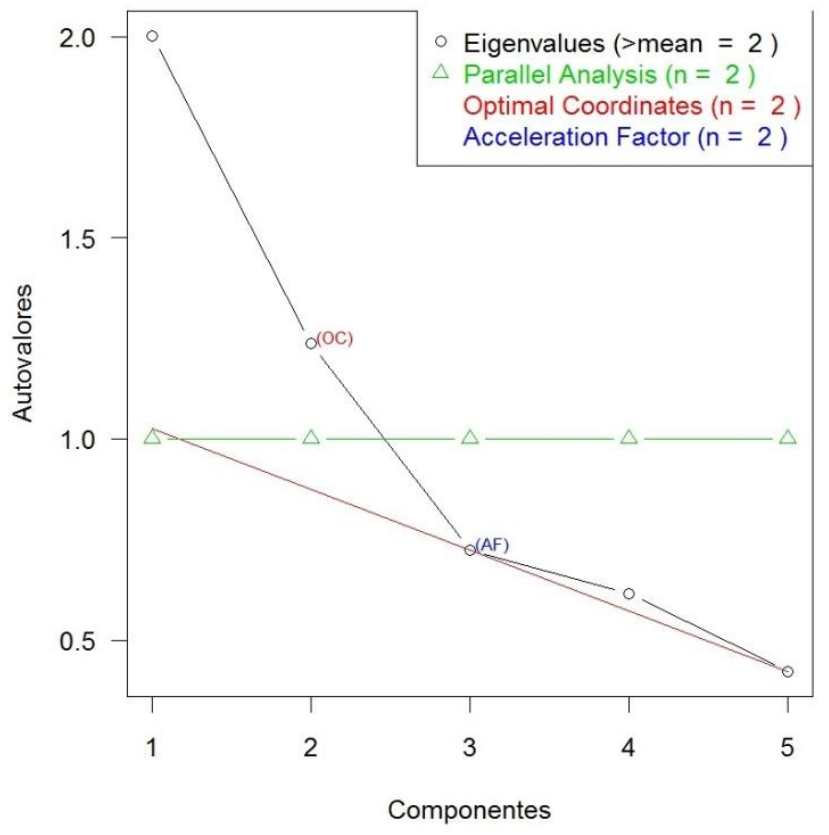

Figura 3. Gráfico scree-plot para as variáveis de produção. 


\subsubsection{Interpretação e Nomeação dos Fatores}

A tabela 3 apresenta a matriz fatorial sem rotação das variáveis de produção, obtida por meio do método dos componentes principais. A tabela 4 apresenta a matriz fatorial com rotação Varimax das variáveis de produção. O objetivo da rotação Varimax é simplificar a matriz maximizando as cargas fatoriais à apenas um fator para uma melhor interpretação dos fatores.

Tabela 3. Matriz fatorial sem rotação das variáveis de produção.

\begin{tabular}{lcl}
\hline VARIÁVEIS & FATOR 1 & FATOR 2 \\
\hline $\begin{array}{l}\text { Bovinocultura } \\
\text { corte }\end{array}$ & 0,81 & 0,11 \\
\hline $\begin{array}{l}\text { Bovinocultura } \\
\text { mista }\end{array}$ & $-0,27$ & 0,74 \\
\hline Bubalinocultura & $-0,19$ & 0,78 \\
\hline Caprinocultura & 0,73 & 0,17 \\
\hline Ovinocultura & 0,83 & 0,16 \\
\hline
\end{tabular}

Tabela 4. Matriz fatorial com rotação pelo critério Varimax das variáveis de produção.

\begin{tabular}{|c|c|c|}
\hline VARIÁVEIS & FATOR 1 & FATOR 2 \\
\hline $\begin{array}{l}\text { Bovinocultura de } \\
\text { corte }\end{array}$ & 0,82 & $-0,09$ \\
\hline $\begin{array}{l}\text { Bovinocultura } \\
\text { mista }\end{array}$ & $-0,08$ & 0,79 \\
\hline Bubalinocultura & 0,01 & 0,81 \\
\hline Caprinocultura & 0,75 & $-0,02$ \\
\hline Ovinocultura & 0,85 & $-0,05$ \\
\hline
\end{tabular}

Uma nomeação possível e condizente para o fator 1, seria "Atividades Produtivas de
Corte". Dessa forma, denominamos o fator 2 de "Atividades Produtivas de Leite".

\subsection{Aplicações das Técnicas nas Variáveis de Número de Unidades de Produção Agrícola}

A tabela 5, apresenta os valores do teste MSA para as variáveis de número de UPA. A tabela 6 apresenta os valores correspondentes aos autovalores para as variáveis de número de UPA.

Tabela 5. Aplicação do teste MSA nas variáveis de número de UPA.

\begin{tabular}{lll}
\hline \multicolumn{2}{l}{ VARIÁVEIS } & VALORES \\
\hline $\begin{array}{l}\text { № UPA com } \\
\text { bovinocultura de corte }\end{array}$ & 0,69 \\
\hline $\begin{array}{l}\text { № UPA bovinocultura } \\
\text { mista }\end{array}$ & 0,40 \\
\hline $\begin{array}{l}\text { № UPA } \\
\text { bubalinocultura }\end{array}$ & com 0,65 \\
$\begin{array}{l}\text { № UPA } \\
\text { ovinocultura }\end{array}$ & com & 0,69 \\
$\begin{array}{l}\text { № UPA } \\
\text { caprinocultura }\end{array}$ & com & 0,54 \\
\hline
\end{tabular}

Tabela 6. Lista de autovalores para as variáveis de número de UPA.

\begin{tabular}{lll}
\hline & AUTOVALORES & VARIÂNCIA \% \\
\hline 1. & 2,12 & 42,44 \\
\hline 2. & 1,12 & 22,50 \\
\hline 3. & 0,80 & 15,96 \\
\hline 4. & 0,53 & 10,57 \\
\hline 5. & 0,43 & 8,53 \\
\hline
\end{tabular}


Analisando o critério da raiz latente considera-se os dois primeiros valores como significativos e excluí-se os demais valores. Analisando o critério de percentagem da variância considera-se que os dois primeiros valores possuem $64,94 \%$ da variância explicada e descarta-se os demais valores.
O critério do teste de scree na Figura 5 mostra o ponto onde os valores obtidos para cada autovalor tende a se estabilizar, então o número de autovalores anteriores a esse ponto, será o número de fatores.

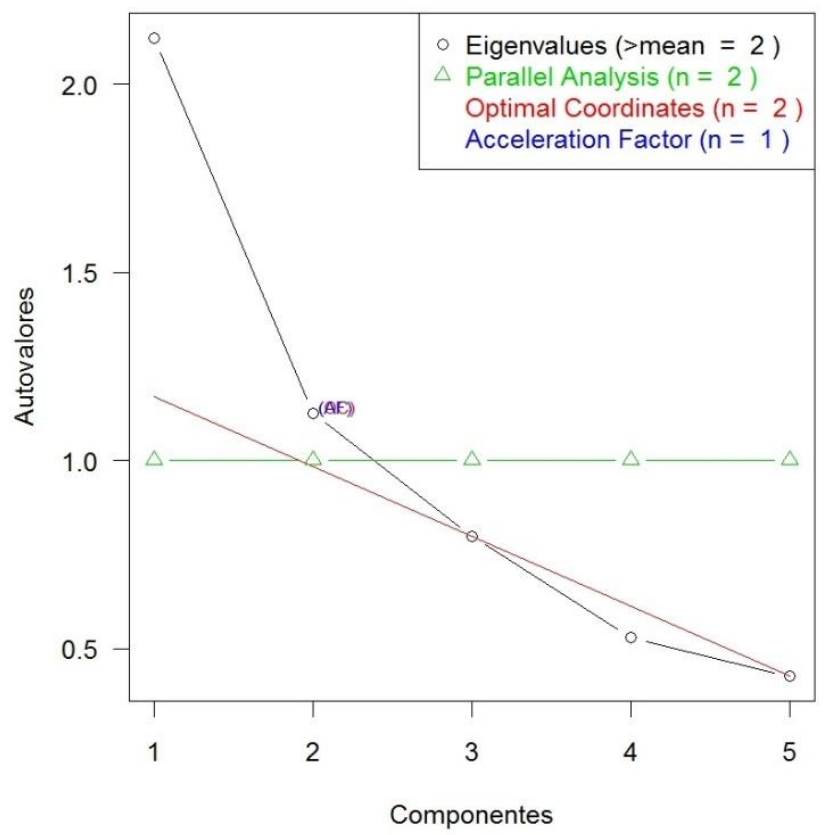

Figura 4. Gráfico scree-plot para as variáveis de número de UPA.

\subsubsection{Interpretação e Nomeação dos Fatores}

A tabela 7 apresenta a matriz fatorial das variáveis de número de UPA, obtida através do método dos componentes principais. A tabela 8 mostra a matriz fatorial com rotação Varimax. 
Tabela 7. Matriz fatorial sem rotação das variáveis de número de UPA.

\begin{tabular}{lclc}
\hline VARIÁVEIS & & FATOR 1 & FATOR 2 \\
\hline $\begin{array}{l}\text { No UPA } \\
\text { bovinocultura } \\
\text { corte }\end{array}$ & $\begin{array}{r}\text { com } \\
\text { de }\end{array}$ & 0,75 & 0,16 \\
\hline $\begin{array}{l}\text { No UPA } \\
\text { bovinocultura mista }\end{array}$ & com 0,08 & $-0,87$ \\
\hline $\begin{array}{l}\text { No UPA com } \\
\text { bubalinocultura }\end{array}$ & 0,81 & 0,23 \\
\hline $\begin{array}{l}\text { No UPA com } \\
\text { caprinocultura }\end{array}$ & 0,78 & 0,07 \\
\hline $\begin{array}{l}\text { No UPA com } \\
\text { ovinocultura }\end{array}$ & $-0,54$ & 0,54 \\
\hline
\end{tabular}

Tabela 8. Matriz fatorial com rotação pelo critério Varimax das variáveis de número de UPA.

\begin{tabular}{lccc}
\hline VARIÁVEIS & & FATOR 1 & FATOR 2 \\
\hline $\begin{array}{l}\text { No UPA } \\
\text { bovinocultura } \\
\text { corte }\end{array}$ & $\begin{array}{r}\text { com } \\
\text { de }\end{array}$ & 0,76 & 0,04 \\
\hline $\begin{array}{l}\text { No UPA com } \\
\text { bovinocultura mista }\end{array}$ & $-0,15$ & 0,86 \\
\hline $\begin{array}{l}\text { № UPA com } \\
\text { bubalinocultura }\end{array}$ & 0,84 & $-0,02$ \\
\hline $\begin{array}{l}\text { No UPA com } \\
\text { caprinocultura }\end{array}$ & 0,77 & 0,14 \\
\hline $\begin{array}{l}\text { No UPA } \\
\text { ovinocultura }\end{array}$ & & & \\
\hline
\end{tabular}

Uma nomeação possível e condizente para o fator 1 , seria "№ UPA com Produções Específicas". Dessa forma, denominamos o fator 2 de "№ UPA com Produções Diversas".

\subsection{Análise de Agrupamento}

Foram divididos de acordo com sua similaridade os municípios da RA de Presidente Prudente em quatro grupos.

- Grupo 1: Pracinha, Nova Guataporanga, Estrela do Norte, Santo Expedito, Sagres, São João do Pau D’Alho, Alfredo Marcondes, Flora Rica, Indiana, Tarabai, Ribeirão dos Índios, Santa Mercedes, Mariápolis, Panorama, Inúbia Paulista, Emilianópolis, Salmourão.

Grupo 2: Anhumas, Paulicéia, Euclides da Cunha Paulista, Monte Castelo, Álvares Machado, Irapuru, Piquerobi, Lucélia, Regente Feijó, Ouro Verde, Caiabu, Osvaldo Cruz, Adamantina, Presidente Venceslau, Caiuá, Santo Anastácio, Pirapozinho, Taciba, Pacaembu, Rosana e Tupi Paulista.

- Grupo 3: Martinópolis, Florida Paulista, Presidente Prudente, Presidente Epitácio, Teodoro Sampaio, Sandovalina, lepê, Nantes, Narandiba, Junqueirópolis, Mirante do 
Paranapanema, Presidente

Bernardes, Dracena e Marabá

Paulista.
- Grupo 4: Rancharia.

Os agrupamentos são apresentados no mapa de agrupamento da Figura 6.

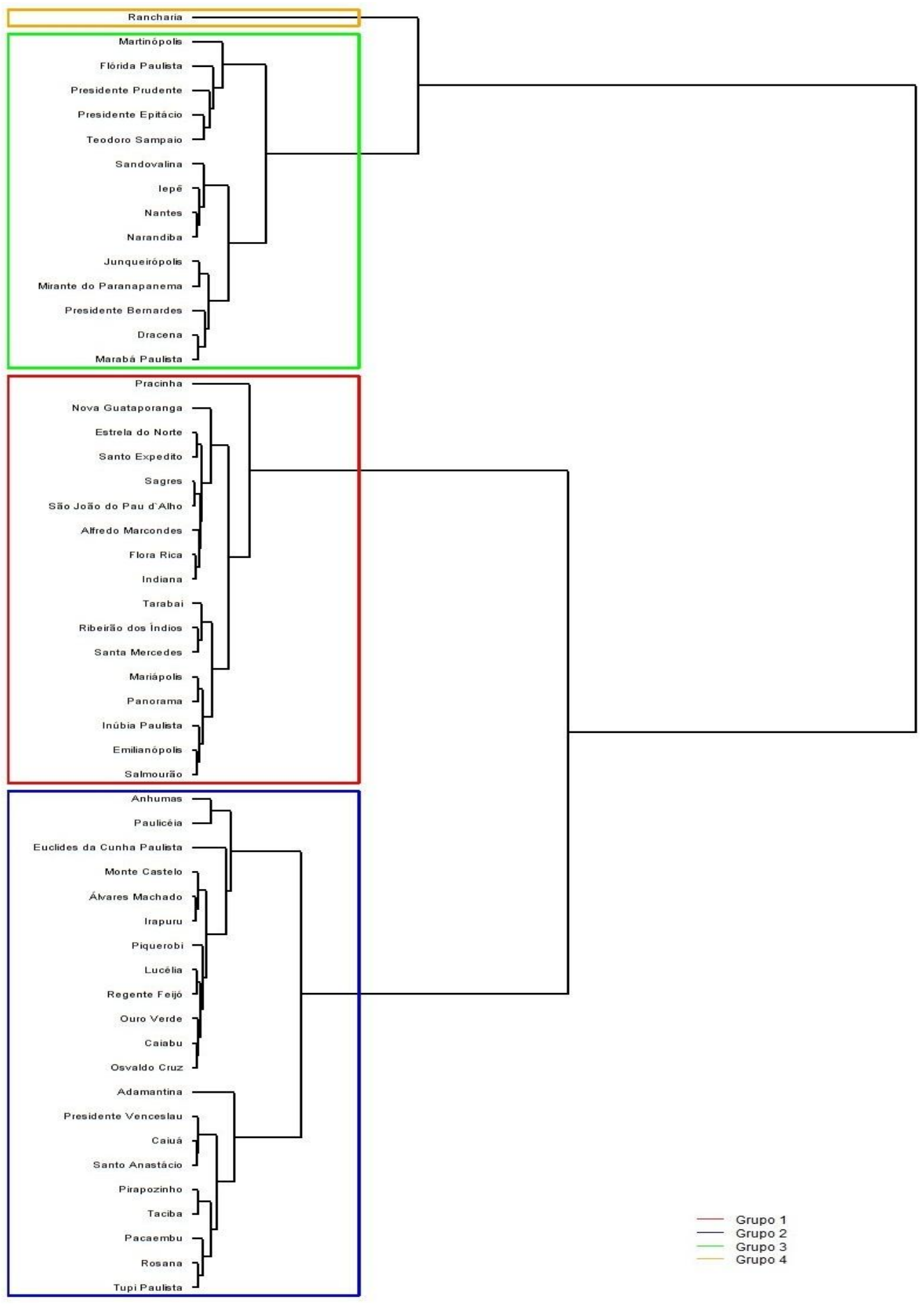

Figura 5. Dendograma. 


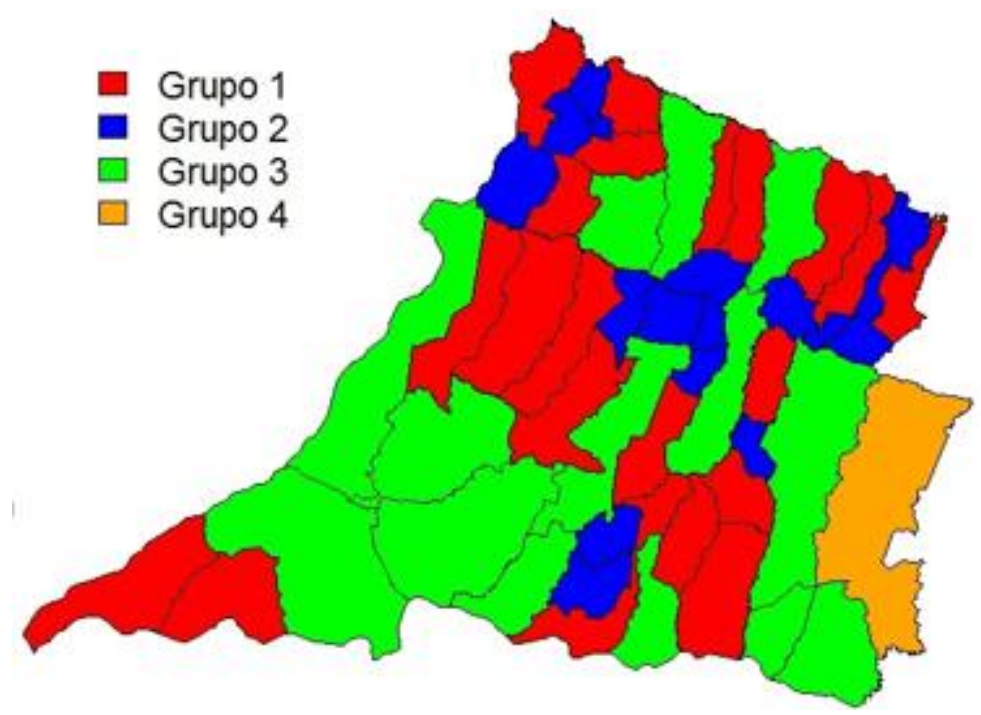

Figura 6. Mapa de agrupamentos.

\section{DISCUSSÃO}

Os resultados mostram que a produção de bovinos de corte e o número de UPA de ovinos contribui significativamente para a economia da região, existindo uma alta correlação entre as variáveis.

Analisando os gráficos de agrupamento observa-se que somente o município de Rancharia compõe o grupo 4, pois Rancharia se destaca por possuir o maior rebanho comercial de bovinos destinados a corte e também a maior contribuíção em relação ao valor adicionado da agropecuária em 2008, da região.

Se sabe que Adamantina tem uma produção significativa de bubalinos, mas também os resultados mostram que Euclides da Cunha Paulista possui alta correlação com o número de propriedades destinadas à produção de bubalinos.

Pracinha, por ser um município muito pequeno e bastante diversificado em sua produção, se mostra importante na bovinocultura mista. Com relação ao número de propriedades destinadas à bovinocultura mista, Anhumas contribui significativamente.

Um resultado a se destacar está relacionado com o município de Martinópolis que apresentou alta correlação com a produção de caprinos.

O grupo 3 possui municípios maiores, em extensão territorial, quando comparado aos grupos 1 e 2. No grupo 3 estão os municípios de Presidente Prudente que de acordo com o gráfico 3 é o município que mais possui propriedades destinadas a produção de bovinos de corte; Marabá Paulista, Presidente 
Epitácio e Martinópolis também de acordo com o gráfico 3 possuem maior correlação com a bovinocultura de corte; já os municípios de Dracena e Junqueirópolis ainda de acordo com a figura 2 possuem maior correlação com a ovinocultura. Percebemos que neste grupo as produções são mais específicas.

No grupo 2 os municípios que se destacam, quando analisamos o gráfico 3, são os municípios de Adamantina que é o município que possui o maior rebanho de bubalinos; Euclides da Cunha Paulista se destaca por ser o município que mais possui propriedades com bubalinocultura e Anhumas qu se destaca por possuir o maior número de propriedades com bubalinocultura mista. Conclui-se quw são municípios que possuem uma extensão de território menor quando comparado ao grupo 3 e em relação à produção, possui uma maior diversificidade.

Apenas Pracinha, grupo 1, possui alta correlação com a bovinocultura mista de acordo com o gráfico 3. Assim podemos concluir que o grupo 1 possui menor extensão territorial quando comparado aos demais grupos e possui maior diversificação em relação à produção.

\section{CONCLUSÃO}

A estrutura produtiva da pecuária de corte na Região administrativa de Presidente Prudente, em sua grande maioria é consideravelmente complexa, uma vez que está baseada em pequenas propriedades com finalidade de subsistência e portando com grande diversidade de produção. Políticas públicas para a região devem levar em consideração esse aspecto no momento da definição de ações que visem à melhoria da eficiência da produção e das condições de vida do trabalhador rural.

\section{REFERÊNCIAS}

FIRETTI, R. et al. Evolução e estrutura da agropecuária no Brasil. Campo Grande: Sociedade Brasileira de Economia, Administração e Sociologia Rural, 2010.

HAIR, J.F. et al. Análise ultivariada de dados. 6. ed. Porto Alegre: Bookman, 2009.

JOHNSON, R.A.; WICHERN, D.W. Applied multivariate statistical analysis. 4. ed. UpperSaddle River: Prentice Hall, 1998.

MINGOTI, S.A. Análise de dados através de métodos de estatística multivariada: Uma abordagem aplicada. Belo Horizonte: UFMG, 2005.

SÃO PAULO (Estado). Fundação Sistema Estadual de Análise de Dados (SEADE). Região administrativa de presidente prudente. Disponível em: <http://www.seade.gov.br/produtos/imp/ind 
ex.php?page=consulta\&action=ano_save $>$.

Acesso em: 24 mar. 2012.

SÃO PAULO (Estado). Levantamento censitário das unidades de produção agropecuária (LUPA). Dados consolidados municipais 2007/2008. Disponível em: <http://www.cati.sp.gov.br/projetolupa/dado smunicipais_xls.php>. Acesso em: 24 mar. 2012.

SILVA, V.M. Os municípios paulistas e o federalismo fiscal brasileiro. São Paulo: IMESP/CEPAM, 1995. 132p. 